\title{
TRL-6 for JWST Wavefront Sensing and Control
}

\author{
Lee Feinberg, Bruce Dean, David Aronstein, Chuck Bowers, Bill Hayden, Rick Lyon, Ron Shiri, \\ Scott Smith \\ NASA Goddard Space Flight Center, Greenbelt, MD 20771 \\ D. Scott Acton, Larkin Carey, Adam Contos, Erin Sabatke, John Schwenker, Duncan Shields, \\ Tim Towell \\ Ball Aerospace \& Technologies Corp., 1600 Commerce St., Boulder, CO 80301
}

Fang Shi

Jet Propulsion Laboratory, California Institute of Technology, Pasadena, CA 91109

Luis Meza

Northrop Grumman Space Technology, One Space Park, Redondo Beach, California 90278

\begin{abstract}
NASA's Technology Readiness Level (TRL)-6 is documented for the James Webb Space Telescope (JWST) Wavefront Sensing and Control (WFSC) subsystem. The WFSC subsystem is needed to align the Optical Telescope Element (OTE) after all deployments have occurred, and achieves that requirement through a robust commissioning sequence consisting of unique commissioning algorithms, all of which are part of the WFSC algorithm suite. This paper identifies the technology need, algorithm heritage, describes the finished TRL-6 design platform, and summarizes the TRL- 6 test results and compliance. Additionally, the performance requirements needed to satisfy JWST science goals as well as the criterion that relate to the TRL-6 Testbed Telescope (TBT) performance requirements are discussed.
\end{abstract}

Keywords: Technology Readiness Level, TRL-6, wave front sensing control, active optics, JWST, space telescope, segmented mirror phasing, phase retrieval

\section{INTRODUCTION}

\section{Science Imaging Requirements}

The James Webb Space Telescope ${ }^{1}$ (JWST) science imaging requirements 2 are based on a series of observation goals that are categorized into various themes. The Science Working Group has developed the Science Requirements Document 3 to describe the objectives implied by these themes for the JWST mission and the performance capabilities necessary to meet these objectives. These science imaging requirements are summarized in Table 1.

Table 1. Image performance metrics ${ }^{3}$ implied by Science Imaging Requirements:

\begin{tabular}{|c|l|}
\hline Parameter & Performance Criterion \\
\hline Strehl Ratio & $\begin{array}{l}\text { Over the FOV of the NIRCam, the observatory shall be diffraction limited at } 2 \mu \mathrm{m} \\
\text { defined as having a Strehl Ratio greater than or equal to } 0.8 .\end{array}$ \\
\hline $\begin{array}{c}\text { Point Spread } \\
\text { Function Stability }\end{array}$ & $\begin{array}{l}\text { The Encircled Energy within a radius of } 0.08 \text { arc-sec at } 2 \text { microns shall not change by } \\
\text { more than } 2.5 \% \text { in less than } 14 \text { days following a worst case slew from a thermal } \\
\text { equilibrium condition at the coldest pointing environment to the hottest pointing } \\
\text { environment. }\end{array}$ \\
\hline
\end{tabular}




\section{Key Technology Driving Requirements}

The three top-level technology objectives derived from the key science imaging requirements are listed in Table 2, as they are allocated to the observatory.

Table 2. Technology Objectives: Key Driving Requirements

\begin{tabular}{|c|l|l|}
\hline Doc \# & \multicolumn{1}{|c|}{ Title } & \multicolumn{1}{c|}{ Requirement Summary } \\
\hline OBS-1607 & Strehl Ratio For Fixed Targets & $\begin{array}{l}\text { Greater than or equal to 0.8 at 2 } \mu \text { m over the NIRCam FOV } \\
\text { (note: met over 14 day worst case slew condition, driving } \\
\text { requirement for WFSC algorithm performance) }\end{array}$ \\
\hline OBS-63 & Image Based Wavefront Sensing & $\begin{array}{l}\text { The Observatory shall perform image-based wavefront sensing } \\
\text { when requested. }\end{array}$ \\
\hline OBS-1733 & Optical Telescope Element FOV & The OTE FOV shall be un-vignetted as shown in Fig. 1. \\
\hline
\end{tabular}

Therefore in order to comply with the JWST scientific objectives, the observatory requires an aperture of 6.6 meter diameter with high sensitivity in the primary band-pass 1.7-30 microns. In addition, a complement of both imaging and multi-field (MF) spectroscopic instruments operating through this band-pass is required and the observatory must be cryogenic $(30-60 \mathrm{~K})$ to minimize observatory thermal background at these wavelengths. The Optical Telescope Element (OTE), composed of a three mirror anastigmatic ${ }^{4}$ telescope design, provides a sufficiently large and well corrected FOV for the instrument complement, with the FOV defined as shown in Fig. 1. The NIRCam, which is part of the ISIM, provides the necessary hardware functionality to create the necessary images needed to perform the image based wavefront sensing by the WFSC algorithms residing on the ground station.

\section{OTE WFE Budget, Rev T}

The TRL- 6 demonstration on the TBT was necessary to prove that the baseline WFSC algorithms are accurate, repeatable, and that they can be used to align the JWST flight TMA telescope. The algorithm success criteria for the demonstration were all confirmed and validated with independent measurements. The success criteria were based on a detailed comparison of Testbed Telescope to flight error budgets and were reviewed in detail with the WFSC Mini-PIT (Performance Integrity Team). The driving goal was showing that a testbed with initial conditions simulating a worst case starting condition on-orbit could be aligned to meet flight WFE requirements over the field of view of the telescope. The Error Budget Traceability section on page 15 gives more details on how the OTE WFE budget was used for TRL- 6 test criteria.

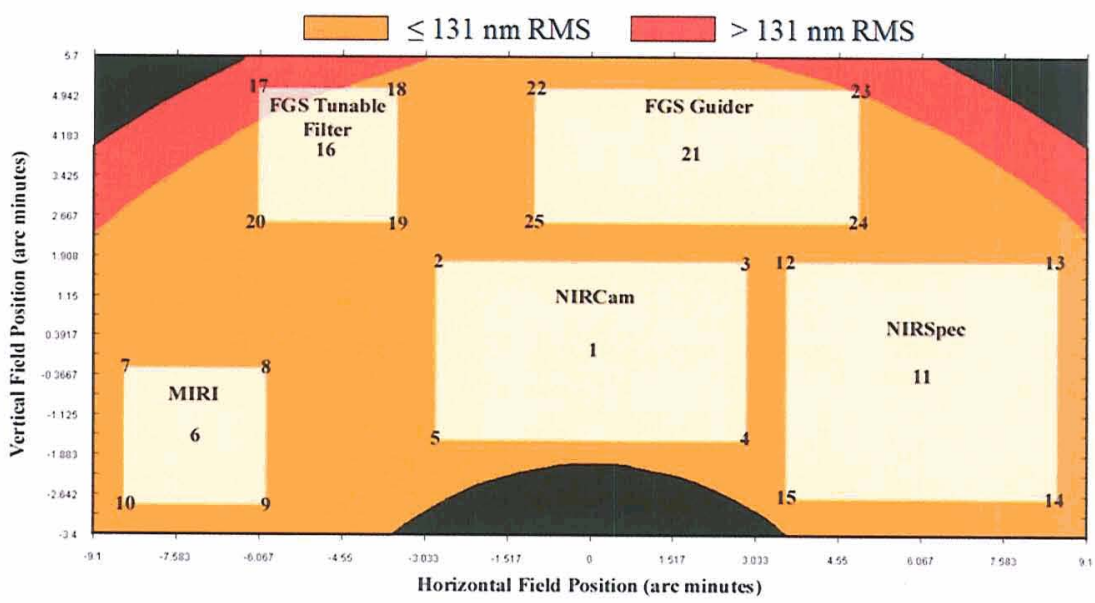

Fig. 1. Optical Telescope Element (OTE) Field of View 


\section{Need for Wavefront Sensing and Control Technology}

In addition to the optical performance requirements of Table 1 and Table 2, the launch platform constraints 5 dictate a segmented primary mirror with an area of $25 \mathrm{~m}^{2}$ and planar density ${ }^{6}$ of less than $25 \mathrm{~kg} / \mathrm{m}^{2}$, and a deployable secondary mirror support structure (SMSS) The characteristics of the JWST primary mirror (hexagonal and segmented primary, with SMSS obscurations, are easily discernible by the pupil geometry shown in Fig. 2 . The primary mirror design is based on a $6.6 \mathrm{~m}, 18$ segment hexagonal array using beryllium ${ }^{7}$ for thermal stability ${ }^{8}$ at the anticipated orbit about the Lagrange Point (L2). As a result of these deployment and thermal stability constraints, the JWST commissioning and periodic optical maintenance must be accomplished using an active optical control system.

During the JWST pre-Phase-A program, extensive modeling efforts were conducted which indicated that an image-based WFSC approach could achieve the required commissioning tolerances using limited control degrees of freedom, with the appropriately allocated deployment accuracy requirements. These telescope deployment as well as other key WFSC requirements are documented in the Wavefront Sensing and Control Requirements Allocation Document ${ }^{9}$ to achieve the observatory commissioning and on-orbit maintenance.

The WFSC subsystem is formed from a set of individual commissioning and maintenance algorithms, along with the associated WFS hardware components required for WFS image

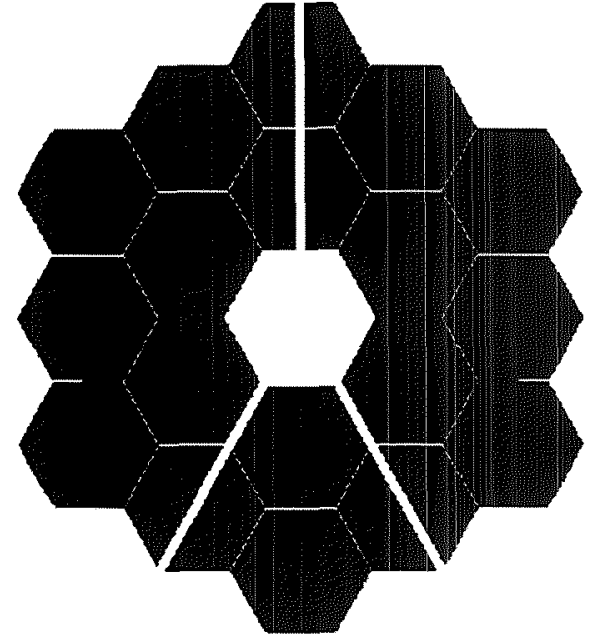

Fig. 2. JWST Entrance Pupil. generation, and the Mirror control algorithm software (MCS) needed for PMSA trajectory and mirror state correction and control. Each algorithm of the WFSC subsystem is listed in Table 3 along with the NASA funded test program used to validate performance. Additional discussion on these test programs is given in the sub-Section entitled "History and Prior Art."

An additional need for the WFSC technology is implied by the nature of the JWST segmented aperture design. Specifically, segments of the primary mirror must be coherently phased with respect to one another in "piston" over the entire aperture. After initial adjustment of the primary mirror during Global Alignment, piston errors are expected to be less than approximately 150 microns (flight) and 30 microns for the Ball TBT (units are surface, PM). For this level of misalignment, a coarse-phasing step (commissioning step \#7 of Table 3) is required to bring the segments within the capture range of the fine phasing algorithms. Coarse phasing has been developed under the JWST test program as a variant of white-light interferometry, by using a Dispersed Hartmann Sensor ${ }^{10}$ (DHS) that is a simple grism-based optical component in the NIRCam filter wheel, with dispersing elements oriented at both zero and 60 degree rotation angles as shown in Fig. 3, in order to provide the data necessary to uniquely solve for all of the segment to segment piston differences.
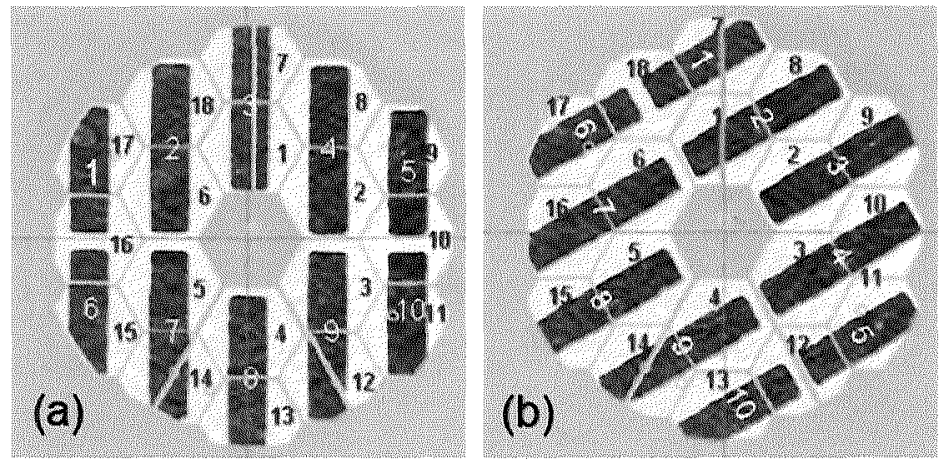

Fig. 3. (a) DHS Element at $0^{\circ}$ Orientation; (b) DHS Element at $60^{\circ}$ Orientation 
Early development work on the DHS component involved in-situ testing on the Keck telescope. In addition, cryogenic testing and vibration testing were also completed as part of the TRL- $6{ }^{11}$ component test process. These results are summarized in Section 3. It should be noted that a complementary piston sensing approach is implemented for the flight system as a backup to the DHS, which is called the Dispersed Fringe Sensor (DFS). 12' 13 The DFS was developed for the JWST Wavefront Control Testbed (WCT) program. Both approaches have been tested on the Keck Observatory segmented telescope system. ${ }^{10,13}$ Early in the program, a trade study 14 was conducted by the JWST project to assess advantages and disadvantages for each of the DHS and DFS methods. It was determined back then that the DHS approach was the best overall method to baseline for flight.

Table 3. JWST WFSC Commissioning Algorithms

\begin{tabular}{|c|c|c|c|}
\hline Algorithm & Purpose & Test Program. & Dates \\
\hline 1. SM focus sweep & $\begin{array}{l}\text { The Secondary Mirror Focus Sweep Algorithm finds an initial } \\
\text { position for the Secondary Mirror (SM). }\end{array}$ & Ball TBT & $2004-2006$ \\
\hline 2. Segment ID & $\begin{array}{l}\text { The Segment Identification Algorithm determines the location } \\
\text { of each of the1 } 8 \text { individual segment images with its associated } \\
\text { Primary Mirror Segment Assembly (PMSA). }\end{array}$ & WCT, Ball TBT & $\begin{array}{l}2000,2004 \\
-2006\end{array}$ \\
\hline 3. Segment search & $\begin{array}{l}\text { The Segment Search Algorithm is used to locate missing } \\
\text { segment images not found during the Segment Identification } \\
\text { process. This algorithm is only performed if there are } \\
\text { segments that were not located in the NIRCam FOV during } \\
\text { segment identification. }\end{array}$ & WCT, Ball TBT & $\begin{array}{l}2000,2004- \\
2006\end{array}$ \\
\hline 4. Image array & $\begin{array}{l}\text { The Segment-Image Array Algorithm moves segment images } \\
\text { into a pre-determined hexagonal image array of the mirror } \\
\text { segments in preparation for Global Alignment }\end{array}$ & Ball TBT & $2004-2006$ \\
\hline $\begin{array}{l}\text { 5. Global } \\
\text { alignment }\end{array}$ & $\begin{array}{l}\text { The Global Alignment Algorithm generates individual } \\
\text { segment wavefront maps that are used to more accurately } \\
\text { position the SM. This algorithm also provides coarse } \\
\text { adjustment corrections for all Primary Mirror (PM) segments. }\end{array}$ & Ball TBT & $2004-2006$ \\
\hline 6. Image stacking & $\begin{array}{l}\text { The Image Stacking Algorithm co-aligns the individual } \\
\text { segment images on top of one another in preparation for } \\
\text { Coarse Phasing. }\end{array}$ & $\begin{array}{l}\text { WCT, Ball TBT, } \\
\text { Ball RA-6 }\end{array}$ & $2001-2006$ \\
\hline 7. Coarse phasing & $\begin{array}{l}\text { The Coarse Phasing Algorithm decreases the Wavefront Error } \\
\text { (WFE) in the PM by adjusting each PMSA piston value. The } \\
\text { Coarse Phasing Algorithm achieves this by correcting segment } \\
\text { piston errors from an } \sim 150 \text { micron level to } \sim 200 \mathrm{~nm} \text {. }\end{array}$ & $\begin{array}{l}\text { WCT, Ball TBT, } \\
\text { Keck Testing, Ball } \\
\text { RA-6. }\end{array}$ & $2000-2006$ \\
\hline $\begin{array}{l}\text { 8a. Fine-phasing } \\
\text { (phase retrieval) }\end{array}$ & $\begin{array}{l}\text { The Fine-phasing Algorithm makes the final adjustments to } \\
\text { the positions of the SM and PM segments to meet the } \\
\text { telescope's WFE performance requirement. } \\
\text { Correct residual segment and SM errors with a sensing } \\
\text { accuracy of } \approx 10 \mathrm{~nm} \text {. }\end{array}$ & $\begin{array}{l}\text { WCT, Ball TBT, } \\
\text { Keck Testing, Ball } \\
\text { RA-6 }\end{array}$ & $1998-2006$ \\
\hline $\begin{array}{l}\text { 8b. Multi-field } \\
\text { fine-phasing }\end{array}$ & Fine align the SM. & Ball TBT & $2005-2006$ \\
\hline $\begin{array}{l}\text { Monitoring and } \\
\text { Periodic Updates }\end{array}$ & Wavefront maintenance will use $8 \mathrm{a}$ and $8 \mathrm{~b}$ as required. & & \\
\hline
\end{tabular}




\section{History and Prior Art}

Prior to JWST, the state of the art in image-based wavefront sensing was phase retrieval (commissioning step \#8a of Table 3). Phase retrieval was demonstrated at TRL-9 for the diagnosis and repair of the Hubble Space Telescope mirror edge defect through the deployment of COSTAR. ${ }^{15}$ Details documenting the Hubble Space Telescope phase retrieval analysis are discussed in a series of NASA Contract reports which have been summarized in the literature. ${ }^{16}$ The first suggestion of using phase retrieval as a wavefront sensing method for JWST was in $1989,{ }^{17}$ nearly a year before the Hubble launch and deployment.

During the JWST program Phase-A, NASA funded several early test programs designed to accelerate technology development and increase the overall robustness and maturity of the fine-phasing phase retrieval technique as well as other WFSC commissioning steps; it should be noted that "fine-phasing" and "phase retrieval" are synonymous throughout this document. This was due to the importance of the fine-phasing algorithm of Table 3, given that this PR algorithm forms the foundation for commissioning algorithms 1, 5, and 8 . These previous test programs were implemented at NASA GSFC beginning in 1998 with DCATT ${ }^{18}$ and continuing through the WCT ${ }^{19}$ test program (WCT-1, WCT-2, and WCT-3). WCT was funded through the conclusion of the JWST Phase A in 2002.

Beginning in JWST Phase B, Ball Aerospace was under subcontract with Northrop Grumman to develop the Ball Testbed Telescope (TBT). The Ball TBT design is an 18 segment flighttraceable TRL-6 test platform (summarized in Section 2). The JWST program development goal for the TBT was its use in the validation and development of the commissioning steps/algorithms outlined in Table 3. Pre-Phase-B algorithm development and testing by Ball Aerospace was performed on the RA-6 Testbed. ${ }^{20}$

During the Phase-A test programs, NASA investigated several
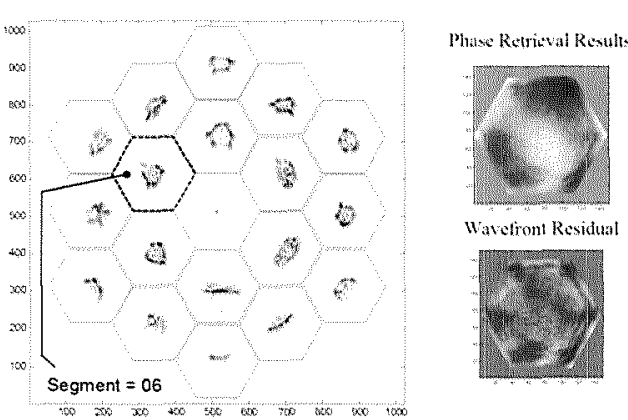

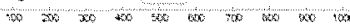
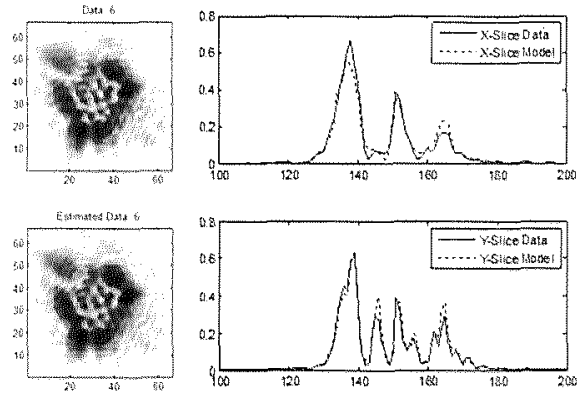

Fig. 4. Extended Dynamic Range HDA Results from TBT First-Light Commissioning.

phase retrieval approaches for JWST. These approaches can be classified into two general categories: iterative-transform, ${ }^{29}, 30,21$ and parametric ${ }^{22,} 23$ methods. Prior to consideration for JWST use, modifications to the original iterative-transform approach ${ }^{30}$ were introduced based on the introduction of a defocus diversity function ${ }^{24}$ or on the input-output method. ${ }^{25}$ Various implementations ${ }^{26}$ of the focus-diverse iterative-transform method have been developed in the prior "state of the art," which deviates slightly from the current implementation by either utilizing a single wavelength or by varying the placement and number of defocused image planes. Finally, modifications to the parametric approach included the inclusion of minimizing alternative merit functions, as well as implementing a variety of nonlinear optimization methods such as Levenburg-Marquardt, simplex, and quasi-Newton techniques. ${ }^{27}$ These techniques and their variants constitute the pre-JWST "state of the art" in phase retrieval.

In summary of the fine-phasing investigations under the JWST program, a strictly parametric approach based on nonlinear optimization was found to be less robust and more sensitive to algorithm starting conditions. ${ }^{28}$ The iterative-transform Misell-Gerchberg-Saxton algorithm, ${ }^{29,}, 30,31,32$ has also been assessed as the earlier Government baseline in the Phase-A Program. It was later determined during initial commissioning work using the TBT in October of 2005, that the Misell-Gerchberg-Saxton approach was found to be insufficient for capturing multi-wave segment wavefronts, which can occur after JWST launch and initial deployment, but prior to the coarse-phasing process being completed (step \#7 of Table 3). Earlier work had suggested that a Modified-Gerchberg-Saxton (MGS) approach could be utilized for multi-wave phase recoveries, but only with an additional phase-unwrapping ${ }^{33}$ post-processing step that leads to a more complicated algorithm procedure which is undesirable for flight. This additional post processing step was also found to be less robust, when compared to an algorithm approach that incorporates feedback through an adaptive diversity function. The Misell-Gerchberg-Saxton algorithm was further developed into the Hybrid Diversity Algorithm (HDA) ${ }^{34}$ as a direct result of the JWST funded programs. Examples of early algorithm results from the first-light commissioning work on the TBT are shown in Fig. 4. The HDA 
approach addresses the need for a large dynamic range (multi-wave) sensing and also the ability to properly sense piston errors of the JWST segmented primary mirror. It is worthwhile mentioning that in order to increase the scope of applicability and robustness of the JWST fine-phasing approach, the HDA and Misell-Gerchberg-Saxton algorithms have been tested using a variety of ground and space-flight projects.

Additional "lessons learned" from the Hubble problem have been incorporated into the JWST WFSC subsystem design, including a pupil-imaging subsystem ${ }^{35}$ to avoid the necessity of estimating pupil amplitude in addition to the pupil phase. This problem was solved for Hubble using the iterative transform approach, ${ }^{36}$ but ultimately led to larger numerical uncertainties than were necessary on the final determination of the primary conic constant. It should be noted that the commissioning steps requiring PR is facilitated by the NIRCam optical design, which includes special filters and WFSC optical components such as weak lenses to generate diversity defocus. Finally, during the course of the JWST technology development program the algorithm implementation details including diversity selection have been resolved, ${ }^{37}$ which were not available during the days of HST.

\section{Scope of the TRL-6 Test Program and Success Criteria}

In order to demonstrate WFSC TRL-6, five key technology elements were identified to be demonstrated (Table 4). These are: (1) development of the DHS component, (2) development of the Coarse Phasing process that incorporates the DHS, (3) end-to-end single-field Fine-Phasing, (4) independent validation of the Fine-Phasing performance, and (5) demonstration and validation of the MF Fine-Phasing process. To help establish these criteria as well as continually review progress, an independent peer review panel, or mini-PIT (Performance Integrity Team), was established by the JWST Project. Discussion and rationale of these results are given in Section 3.

In terms of optical hardware, NIRCam ${ }^{38}$ is the imaging system used on Flight for collecting the focal plane image data used as input to each of the commissioning steps in Table 3. Verification of the specific optical design implementation of NIRCam is not within the scope of the TRL-6 test program, since the specific technologies requiring TRL-6 validation are the algorithm commissioning steps listed in Table 3. Validation of the NIRCam optical performance and ability to function as the JWST wavefront sensor has been assessed in separate design reviews, and will be tested in hardware during both the NOTES and OSIM test programs using JWST specific phase-plates (18 segments) inserted into the NIRCam / NOTES pupil. In addition, TRL-6 verification of specific opto-mechanical interfaces such as the nanometer level step actuators and pre-flight control of the PMSA hexapod interface to the individual mirror segments are being accomplished under separate AMSD and PMSA test programs. Therefore, the wavefront sensing commissioning steps by themselves provide and address the WFSC core technology that is needed to support the new science capabilities enabled by JWST. This distinction limits the scope of the TRL- 6 test program to the algorithms / commissioning steps of Table 3. The 5 technology items that form the TRL-6 success criteria are summarized in Table 4.

Table 4. TRL-6 Compliance Includes 5 Key Demonstrations

\begin{tabular}{|c|c|c|}
\hline Function & Success Criteria & Test Location \\
\hline DHS Element & $\begin{array}{l}\text { - random vibration, } 3 \text { axis, } 1 \text { hour each axis } \\
\text { - thermal cycles from } 300 \mathrm{~K} \text { to } 30 \mathrm{~K} \\
\text { - No degradation in optical performance }\end{array}$ & Element Testing \\
\hline Coarse Phasing & $\begin{array}{l}-18 \text { Keck segments with large piston errors; close-loop } \\
\text { phased to "near" Keck's PCS measured piston } \\
-18 \text { TBT segments phased to capture range of phase } \\
\text { retrieval }\end{array}$ & $\begin{array}{l}\text { Keck Experiment } \\
\text { Testbed Telescope }\end{array}$ \\
\hline $\begin{array}{l}\text { End to End Single- } \\
\text { Field Fine-Phasing }\end{array}$ & $\begin{array}{l}\text {-End-to-end performance using all algorithms } \\
\text {-Achieve a final WFE, } \\
\text { (median of delta WFE between test results) } \\
\text {-Show good convergence seven times }\end{array}$ & Testbed Telescope \\
\hline $\begin{array}{l}\text { Phase Retrieval } \\
\text { Performance }\end{array}$ & $\begin{array}{l}\text { - includes actual environmental drift, double pass compared } \\
\text { to IF reference over controllable modes }\end{array}$ & Testbed Telescope \\
\hline
\end{tabular}




\begin{tabular}{|c|c|c|}
\hline $\begin{array}{c}\text { Multi-Field Fine- } \\
\text { Phasing }\end{array}$ & After correcting field-dependent aberration & Testbed Telescope \\
\hline
\end{tabular}

\section{TRL-6 TEST PLATFORM: THE TBT TESTBED TELESCOPE}

\section{Overview}

The JWST WFSC TRL-6 test platform is a meter-class 18 segment Testbed Telescope (TBT). ${ }^{39}$ Precursor technology development for the TBT began with the Ball RA-6 Testbed. ${ }^{20}$ The TBT is a complex electro-optical system consisting of a flight traceable optical design with an optical trunk circuit and a three mirror anastigmat Optical Telescope Element (OTE). ${ }^{40}$ The trunk circuit provides access to the system pupil, and emulates the functionality of the NIRCam. Like JWST, the TBT primary mirror consists of 18 segments that each incorporate Primary Mirror Segment Assembly (PMSA) actuator control in a flight-traceable design, under the constraints of tighter packaging requirements caused by the 1-meter aperture design. The optical layout and primary mirror of the TBT is shown in Fig. 5.
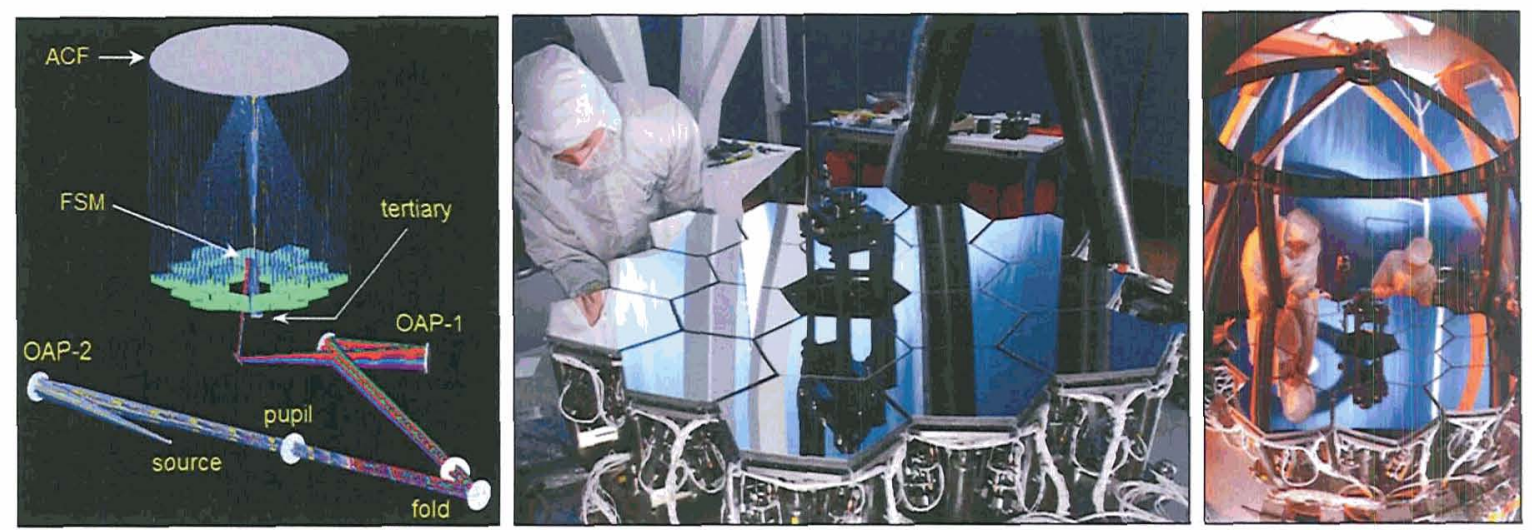

Fig. 5. TBT Optical Layout and Primary Mirror.

Based on the flight optical model, each segment position error causes a pre-determined effect in the wavefront or image plane. The TBT has been designed such that the analogous perturbation generates an analogous effect on a TBT segment. Scaling differences were determined based on a variety of miscellaneous TBT physical size differences and constraints such as shorter focal length, different wavelengths, and image plane size.

\section{TBT Environmental Factors}

Environmental factors in the TBT laboratory are comprised of 3 terms: jitter, air turbulence, and drift. Each term has been characterized and included in the uncertainty error budgets that were used to establish the TRL-6 test criteria. ${ }^{41,42}$ The jitter is comprised of seismic and acoustic inputs which have been mitigated in the TBT optomechanical design. Seismic inputs are attenuated by using 7 Newport pneumatic isolation towers supporting the massive Large Optical Test Facility (LOTF). Acoustic inputs were reduced by removing large flat structural pieces not needed for the LOTF stability. Air turbulence is diurnal and can have a significant optical affect due to the long optical path length of approximately 28 meters. ${ }^{43}$ Drift is primarily thermally driven and manifests itself mostly in the bending of the "wings" of the primary mirror; each mirror wing has 3 segments. Additionally, air temperature sensors near the TBT were used to identify "quiet" times. Thermally quiet times have been necessary for most of the commissioning tests.

\section{Detectors used for TBT Commissioning}

The TRL-6 implications for the fine-phasing algorithm are to produce wavefront estimates from focal plane data ${ }^{44}$ that scale to the expected JWST detector sampling, dynamic range (bit depth), quantization, pixel modulation 
transfer function (MTF), and signal-to-noise ratio (SNR) of the expected flight system images. To observe this level of detector and image-data traceability, the TBT fine-phasing camera, like the flight detector for NIRCam, is based on a $\mathrm{HgCdTe}$ detector array.

The NIRCam focal plane array is a unique imaging device that offers low noise, high resolution IR imagery over a large field of view. Since it is not practical to reproduce the exact NIRCam focal plane array on the TBT, two separate cameras were utilized to emulate the functionality of NIRCam, while emulating (as close as possible) key phase retrieval sampling parameters that are specified for flight. The first camera, which is a visible camera, is the Princeton Instruments MicroMAX CCD system. This camera has a large detector FOV comparable to a single channel of NIRCam. The visible camera is incorporated into the commissioning process where a large FOV is required, but high resolution is not. The second camera is the CEDIP Infrared Systems JADE short-wave IR imager. This camera has a relatively small FOV, and supports part of the commissioning process that requires highresolution imaging in the IR. Given that the JADE camera is based on a $\mathrm{HgCdTe}$ detector array, it is expected that this camera will have performance characteristics that are traceable to that of NIRCam flight array. However, the actual NIRCam camera is expected to collect data sets of much higher quality as can be seen by comparing detector characteristics such as Read Noise, Dark Current, Quantum Efficiency, and Well Depth. Given that the flight data sets are expected to be of higher quality in terms of SNR, we similarly expect the WFSC results to be of higher fidelity and quality when compared to the TBT results. WFS tolerancing as a function of these noise parameters and other model mismatch errors, specific for JWST flight, has been published in the literature. ${ }^{45}$ Recent tolerancing work for the ISIM imaging system in support of OSIM activities has been presented to the JWST project. ${ }^{46}$

\section{Error Budget Traceability}

As described in the Section "OTE Error Budget," the Post WFSC box in the OTE budget was used in the determination of the TRL- 6 test criteria. The TRL- 6 criteria also factored in single vs. double pass requirements (as appropriate) and environmental factors both in flight and on the ground. The detailed comparisons were documented at the September WFSC Mini-PIT ${ }^{42}$ meeting and reviewed by that panel. The panel did make recommendations relative to the multi-field criteria which were then documented in the multi-field whitepaper. ${ }^{53}$ Though TRL-6 criteria were derived by lower level error budget comparisons, the total telescope WFE of the flight and testbed telescopes are comparable and the allocations for performance are smaller than the RSS of the actual error budgets and the reserve in the OTE budget. This gives us good confidence that by meeting the detailed criteria set out for TRL- 6 , we can meet the flight requirements with margin.

\section{TBT Optical Performance after Realignment}

When initially placed on the TBT backplane, the travel ranges of several individual mirror hexapods were insufficient to align the TBT segments to their WFSC predicted positions. Therefore, in August 2006, the mini-PIT recommended that the PM segments should be realigned by radially re-positioning the segments to their minimum wavefront predicted positions, as predicted by the WFSC system. As a result of this realignment, the hexapod adjustment ranges for the ROC degree of freedom, in addition to the radial and clocking degrees of freedom (enabling astigmatism control), are now within the range of actuator movement margins, and centered about the actuator travel limits.

\section{RESULTS FROM THE TBT TELESCOPE}

\section{Overview}

In this Section, the five key technology elements are demonstrated that were listed earlier in Table 4. As discussed earlier these are

1. Development of the DHS component

2. Development of the Coarse Phasing process that incorporates the DHS

3. End-to-end single-field point Fine-Phasing (demonstrate repeatability of $55 \mathrm{~nm}$ RMS for the median of subtracted wavefronts) 
4. Independent validation of the Fine-Phasing performance (comparison of single-field phase retrieval to interferometry should be $76 \mathrm{~nm}$ RMS)

5. Demonstration and validation of the MF point Fine-Phasing process (performance of 60nm RMS over 4 field corners)

To help establish these criteria as well as continually review progress, an independent peer review panel, or PIT (Performance Integrity Team), was established by the JWST Project. The review panel consists of experts in various JWST telescope disciplines. The mini-PIT ${ }^{47}$ is a smaller peer review panel of experts in optics and WFSC techniques. During the course of reviews and periodic presentations to the mini-PIT panel, the WFSC team at Ball made significant progress on establishing results in support of the five technology elements listed above (and in Table 4). An overview of the results of meeting these five criteria are presented in the following sections.

\section{Development of the DHS Component}

The Technology Readiness of the DHS Coarse Phasing Sensor, as a hardware component, was evaluated through extensive testing of a Technology Readiness Demonstration (TRD) test article in the fall of 2004 . Although cryogenic grisms have been flown successfully on several previous missions, the DHS element is unique in that it requires small grism patches to be bonded to a single element. Thus, the primary goals of the TRD testing were to evaluate the suitability of the flight-like design under vibration and thermal stress testing in a launch environment, while maintaining optical performance. A flight-like article was produced by Adaptive Optics Associates (AOA) ${ }^{48}$ and was thoroughly tested to assess survivability and optical performance. The following tests were conducted on the TRD:

(a) Vibration testing (random, for 1 hour in each axis), thermal cycling between $300 \mathrm{~K}$ and $30 \mathrm{~K}$ (some as low as $10 \mathrm{~K})$,

(b) Optical measurement/verification of the optical performance of the DHS was made at both ambient and at cryogenic temperatures.

(c) The optical testing was followed by microscopic examination of the device, and performance testing using a phase step input to verify the proper measurement of a phase step.

All results were highly successful and as a result of this effort, the DHS element itself was declared to be at TRL-6.

\section{Coarse Phasing Algorithm Testing and Development}

Two technology demonstrations of Coarse Phasing using the Dispersed Hartmann Sensor (DHS) were done at the Keck Observatory in February 2003 and June 2005. The first was a TRL-4 test which demonstrated the feasibility and methodology of dispersed fringe sensing for coarse phasing. The second provided the first TRL- 6 functional test validating the feasibility and methodology of the JWST baseline dispersed fringe sensing process. Fig. 6 is an image of the 10-meter Keck-I Observatory, a world class segmented ground-based telescope.

Adaptive Optics Associates (AOA) custom-built the TRL-6 DHS components that were used on the Keck observatory. These are functionally identical to the flight DHS assemblies and are shown in Fig.

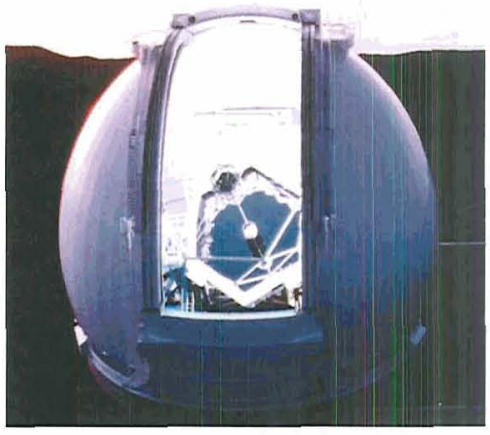

Fig. 6. The Keck I Observatory 7. They were manufactured from a simple grism + Shack-Hartmann mask which creates separated fringe images from 10 inter-segment edges in 2 orientations. The process is identical to the JWST flight implementation and simultaneously determines inter-segment piston differences. The randomized segment pistons were made using bright $\mathrm{K} 0$ and $\mathrm{K} 2$ stars in the presence of atmospheric turbulence. 

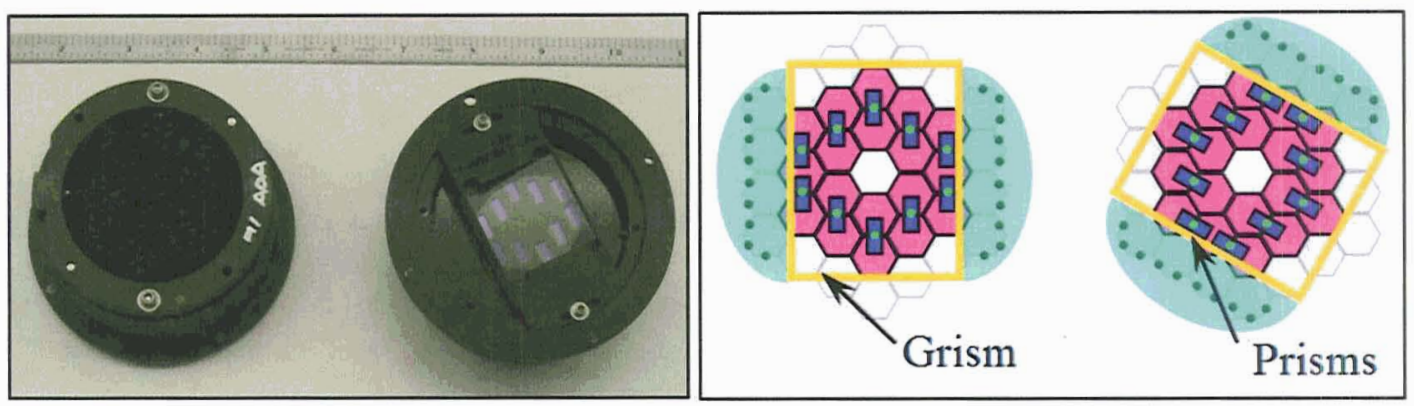

Fig. 7. DHS Assembly for the Phasing Camera System

Fig. 8 shows the 20 DHS fringes for both DHS orientations. The frequency and the angle of the "barber pole" is the measure of piston difference. It was shown that the DHS algorithm results were in good agreement with the established Phasing Camera System (PCS) (Fig. 9) which is a modified Shack-Hartmann camera used at Keck for $10+$ years. The resulting phase error from closed-loop DHS control of the Keck as measured by (PCS) showed good performance (Fig. 10(a)). A statistical comparison of the PCS to the DHS is shown in Fig. 10(b), indicating excellent agreement between DHS and PCS.
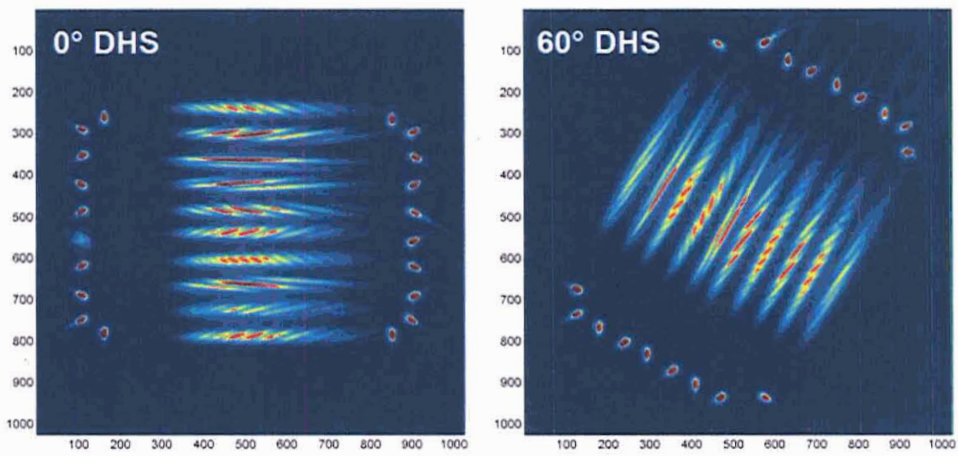

Fig. 8. Keck DHS Fringe Images

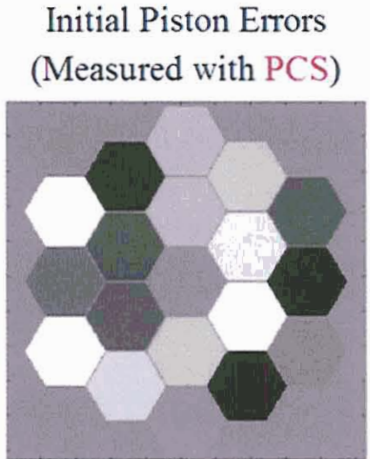

$\operatorname{Min}=-5.96, \operatorname{Max}=5.09 \mu \mathrm{m}$

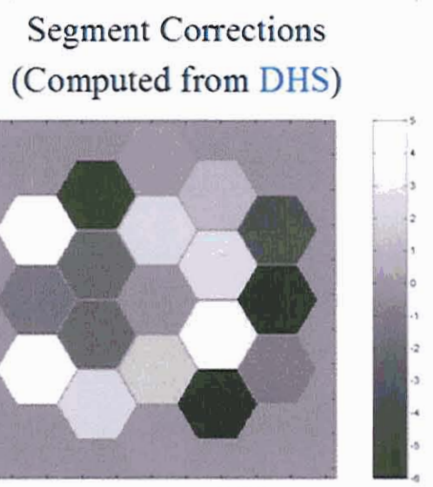

Min=-6.30, Max=5.64 $\mu \mathrm{m}$

Fig. 9. Edge Height Comparison: DHS vs. PCS 

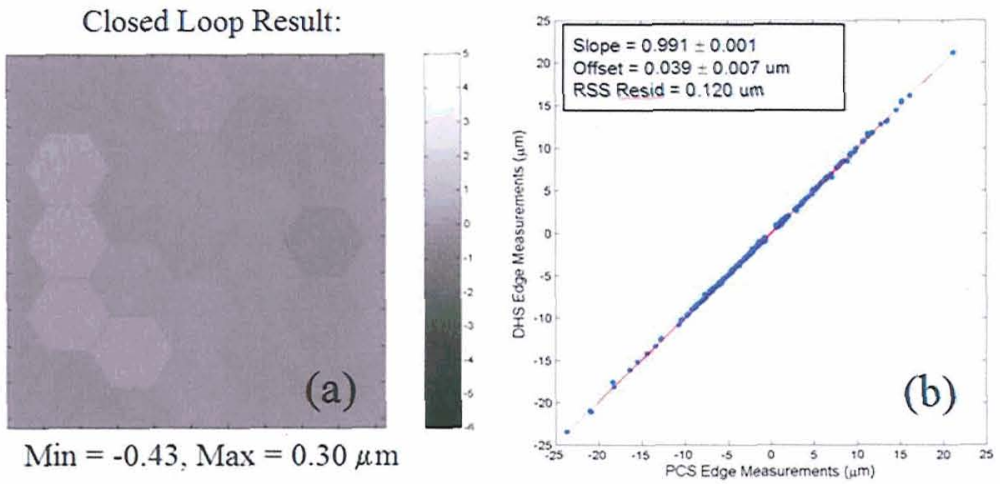

Fig. 10. PCS Measure of DHS Performance

\section{Demonstration of Single-field Point End-End Commissioning}

\section{Overview}

In this Section, an overview of TBT results and data for each commissioning algorithm listed in Table 3 are presented using single-field point image data. A separate paper ${ }^{49}$ in these proceedings describes this data in much more detail. The block diagram ("waterfall" chart) in Fig. 11 illustrates the chronological order of the commission steps. As a point of comparison with the flight implementation, the commissioning steps as implemented on flight and on the TBT are compared in Table 5. Early results from the commissioning process have been documented in the literature. ${ }^{50}$ From Table 9 it is shown that the main differences between flight and the TRL- 6 implementation are isolated to the double-pass nature of the TBT optical design. As a result, it has been necessary to implement a Field Steering Mirror (FSM) to query the WFSC performance over the TBT FOV. This differs from the flight implementation in that the entire observatory will be slewed to image the FOV on orbit. Multi-field phasing results are discussed later in that sub-Section.

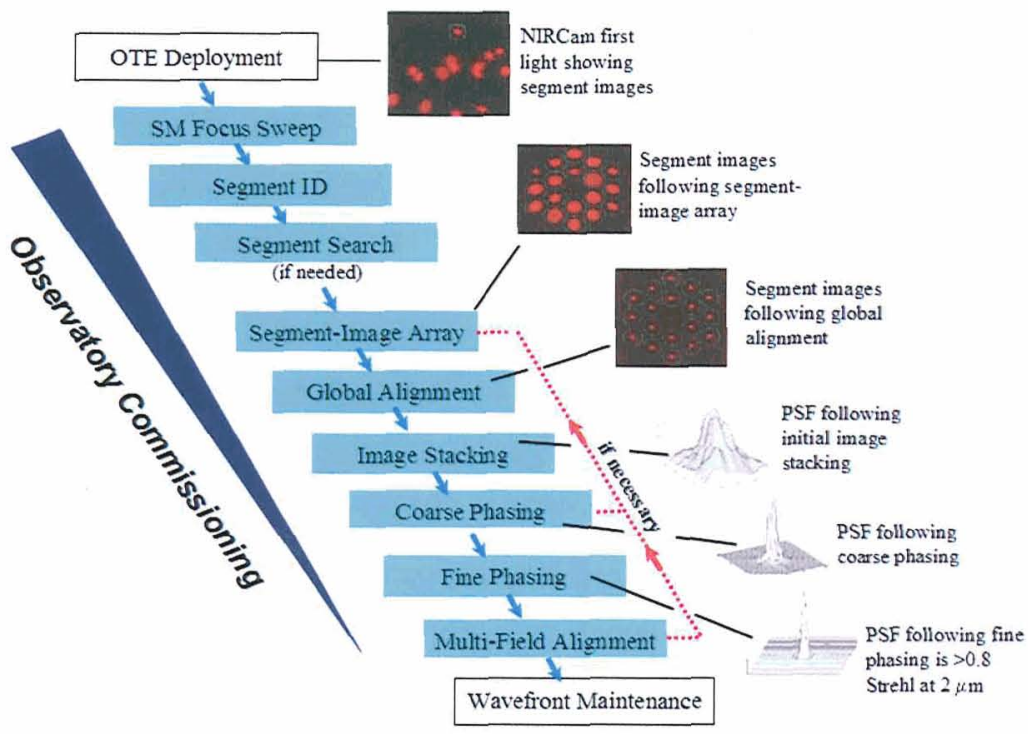

Fig. 11. Block Diagram of Commissioning Steps

The results presented below document the first end-to-end commissioning demonstration on the TBT, which was conducted over a 1-week period starting on October 12, 2006. All steps up through image stacking were performed 
at a wavelength, $\lambda=650 \mathrm{~nm}$ using the wider FOV visible camera. Fine phasing was performed at $1550 \mathrm{~nm}$ using the HgCdTe detector. The image data shown in this report are displayed on a logarithmic scale.

Table 5. Comparison of Flight and TBT Implementation of Commissioning Steps.

\begin{tabular}{|c|c|c|}
\hline Commissioning Steps & Flight & TBT \\
\hline 1. Telescope Focus Sweep & Baseline Process. & Repeat Baseline in Double-Pass. \\
\hline 2. Segment ID & Baseline Process. & Repeat Baseline in Double-Pass. \\
\hline 3. Segment Search & Baseline Process. & Repeat Baseline in Double-Pass. \\
\hline 4. Image Array & Baseline Process. & Repeat Baseline in Double-Pass. \\
\hline 5. Global Alignment & Baseline Process. & Repeat Baseline in Double-Pass. \\
\hline 6. Image Stacking & Baseline Process. & Repeat Baseline in Double-Pass. \\
\hline 7. Coarse Phasing & Baseline Process. & Repeat Baseline in Double-Pass. \\
\hline $\begin{array}{l}\text { 8a. Fine-Phasing } \\
\text { (single-field point) }\end{array}$ & Baseline Process. & Repeat Baseline in Double-Pass. \\
\hline $\begin{array}{l}\text { Verification } \\
\text { (fine-phasing) }\end{array}$ & $\begin{array}{l}\text { Baseline Process: } \\
\text { (a). Scan FOV by Telescope } \\
\text { Slew. } \\
\text { (b). Perform PR. }\end{array}$ & $\begin{array}{l}\text { Repeat Baseline in Double-Pass. } \\
\text { (a) Scan FOV using Field } \\
\text { Steering Mirror (FSM) and ACF } \\
\text { (auto-collimating flat) rather than } \\
\text { Slew. } \\
\text { (b) Perform PR. }\end{array}$ \\
\hline NIRCam FOV: & $\begin{array}{l}\text { If wavefront variation over FOV } \\
\text { is acceptable, skip Step } 8 \text { below. }\end{array}$ & Need to verify on TBT \\
\hline OTE FOV: & $\begin{array}{l}\text { Fine phasing not baselined at each } \\
\text { SI. Verification of performance } \\
\text { over OTE FOV will be indirectly } \\
\text { assessed by performance of SI's. }\end{array}$ & $\begin{array}{l}\text { Verification accomplished using } \\
\text { the Field Steering Mirror (FSM). }\end{array}$ \\
\hline $\begin{array}{l}\text { 8b. Multi-Field Fine- } \\
\text { Phasing. }\end{array}$ & $\begin{array}{l}\text { Baseline: } \\
\text { Perform WFS at } 5 \text { NIRCam field } \\
\text { points. } \\
\text { Derive and implement control. } \\
\text { Note: this step may not be } \\
\text { required for Flight if Step } 5 \\
\text { successful to anticipated accuracy. }\end{array}$ & $\begin{array}{l}\text { Repeat Baseline in Double-Pass. } \\
\text { (a) Scan FOV using Field } \\
\text { Steering Mirror (FSM) and ACF } \\
\text { rather than Slew. } \\
\text { (b) Perform WFSC. }\end{array}$ \\
\hline
\end{tabular}

OTE Deployment conditions

The deployment requirements are documented in an earlier Ball Syst deployment values were randomly generated based on the worse-case flight deployment specs. In deployment cases where the bore-sight of the TBT fell off of the detector, the ACF was adjusted to roughly re-center the deployed segment images on the visible camera. This step is analogous to re-pointing the flight telescope to acquire a first light image. Fig. 12 shows the first-light image after deployment and subsequent repointing of the ACF.

\section{SMFocus Sweep}

The SM Focus Sweep algorithm steps through global focus of the OTE with the secondary mirror (SM) while recording focal plane images at each step. Individual segment images are analyzed with the phase retrieval algorithm to determine the focus error for each segment (relative to the best focus position). The SM is moved as necessary to set the average focus error among the segments to zero.

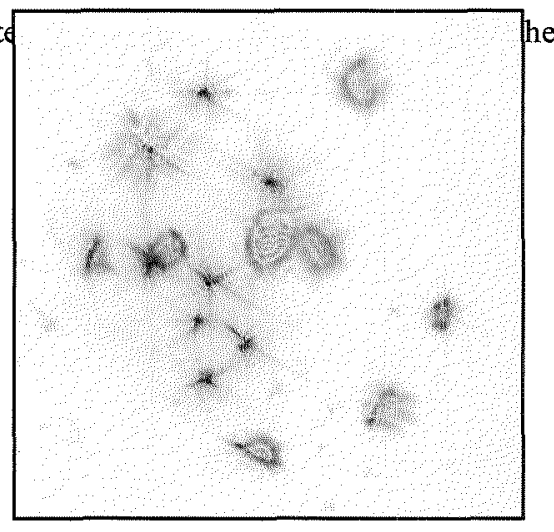

Fig. 12. First Light Deployment Image

Relative SM piston values of $[-100,-50,0,50,100]$ microns were used to support the Focus Sweep in the commissioning demonstration.. The Focus Sweep algorithm estimates the focus error (and an associated SM piston correction) from all images that are clearly visible on the detector. The focus errors are determined through phase retrieval. An average value is applied to the SM as a correction, and a second set of Focus Sweep data is taken and analyzed.

\section{Segment ID and Segment Search}


The segment ID process is the simplest of the commissioning activities. To begin, 19 focal plane images are collected in succession. After each image is collected, small perturbations are applied to individual segments in tip and tilt. From this data, 18 successive image differences are calculated each revealing a moved segment.

In the segment search process, the missing segments are scanned in a spiral pattern, taking an image after each move. A differencing technique determines whether or not the segment image has been detected as discussed above. For the TRL- 6 commissioning demonstration, all TBT segments were identified. Out of 18 segments, 2 of the segments were off of the detector upon initial deployment and were located through the Segment ID and Segment Search process.

\section{Image Array}

After the segment images are all identified, they are moved from their current random locations to an image array based on mathematically generated vertex locations for a hexagonal array. In the specific commissioning demonstration, two iterations were required to position the images.

\section{Global Alignment}

The Global Alignment process analyzes segment-level wavefronts with the phase retrieval algorithm. ${ }^{34}$ From these phase retrieval results SM modes (piston, X-roll, Y-roll) and PMSA modes for each segment (piston, clocking, and radial translation) are controlled. Diversity defocused images that are input to the phase retrieval algorithm, are obtained by changing the SM piston by a known amount. Changing the global defocus in this step also moves the spots within the image array, and consequently, corrective PMSA tip/tilts are applied to maintain the array positions after each move. Two diversity defocus positions are used.

The extracted images were analyzed with a phase retrieval algorithm to recover the segment level wavefronts. The wavefront is dominated by PM segment piston errors (resulting in power terms in the individual segments). Clocking and radial translation errors (seen as astigmatism) are also present. Less obvious are the global errors associated with positioning errors in the SM. The 3 corrective modes for the SM and each PM segment were estimated and applied as corrections.

Image stacking

The goal of the image stacking process is to precisely stack the segment images on top of each other, in preparation for allowing the segments to coherently combine as a phased aperture.

\section{Coarse Phasing}

The piston errors between the individual segments are measured with the pair of DHS masks described in Section 1 (Fig. 3). Relative piston errors were measured on the TBT for each segment, consistent with the expected deployment misalignments. Corrections were then applied and additional DHS images were taken and analyzed.

The accuracy on the DHS piston measurement technique on the TBT was demonstrated in subsequent DHS tests, one of which is illustrated in Fig. 13. These subsequent coarse phasing tests demonstrate that the algorithm is capable of making very accurate PM segment piston measurements, well within the JWST requirements. Successive applications of the coarse phasing algorithm reduced the PM segment piston errors, followed by three additional iterations (Fig. 13) which further reduced the errors. 


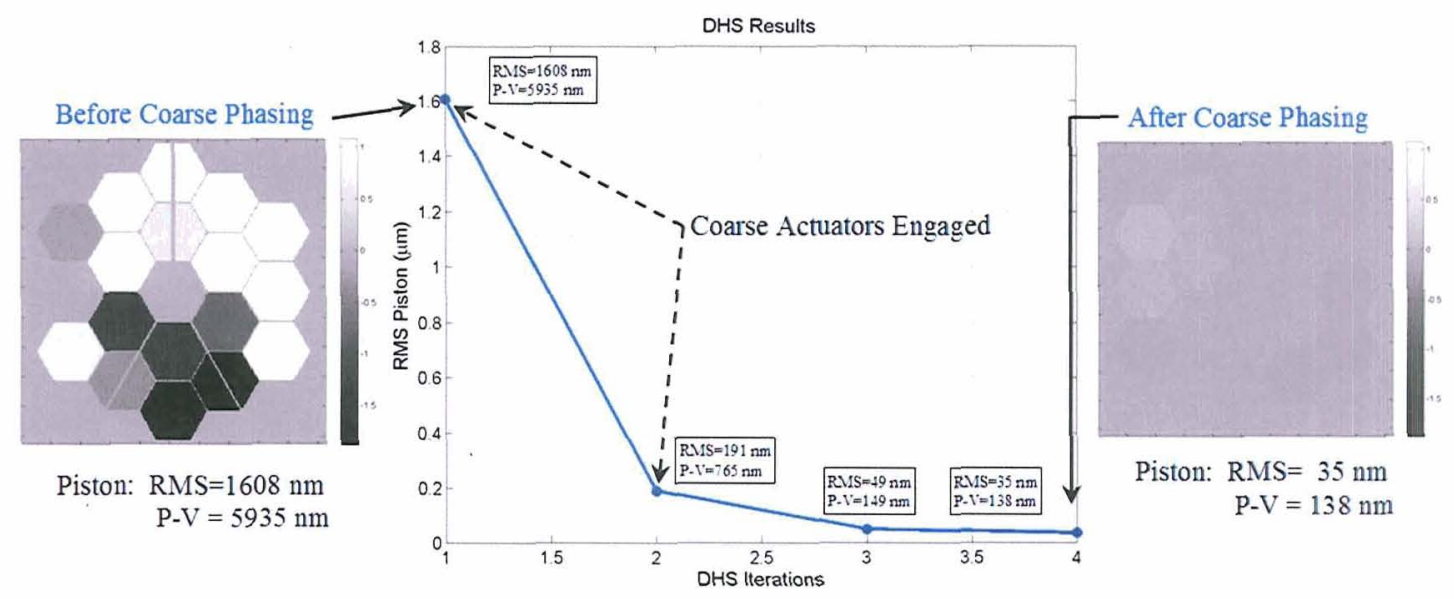

Fig. 13. Additional iterations of the coarse phasing algorithm reduced the PM segment piston errors.

\section{Fine-Phasing}

Immediately following the coarse phasing process, defocused IR images were collected and analyzed using the phase retrieval algorithm. The Fine-Phasing process uses weak lenses to create \pm diversity defocused images (Fig. 14, top row). The images shown in Fig. 14 were then used as input to the phase retrieval algorithm to estimate individual segment tip, tilt, and piston errors, as well as SM de-space. The initial phase retrieval estimate is then used as the starting wavefront ( $0^{\text {th }}$ iteration; Fig. 14, lower left). In both the images and the wavefront, segment level wavefront errors are readily visible as well as an overall SM piston error (focus). The PM and SM moves were derived from the fine-phasing results shown in Fig. 14 and applied as corrections. After repeating this process several more iterations as shown in Fig. 14, nearly all controllable misalignment errors in the wavefront were eliminated (Fig. 14, lower right).

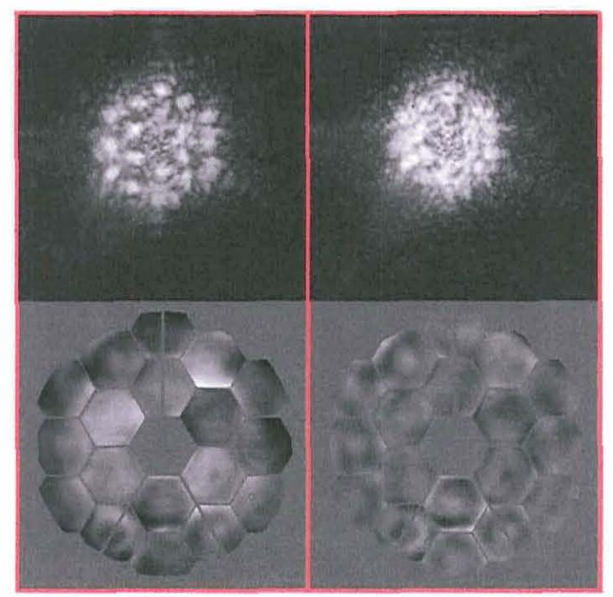

Fig. 14. Initial fine-phasing image and wavefront and after applying corrections.

\section{Fine-Phasing Convergence}

Following the initial end-to-end commissioning demonstration, seven additional fine-phasing tests were conducted (Fig. 15) starting with errors consistent with large but successful completion of the coarse phasing process. In each case, the wavefront errors converged to a wavefront error well within the TRL- 6 single-field point commissioning criteria. 


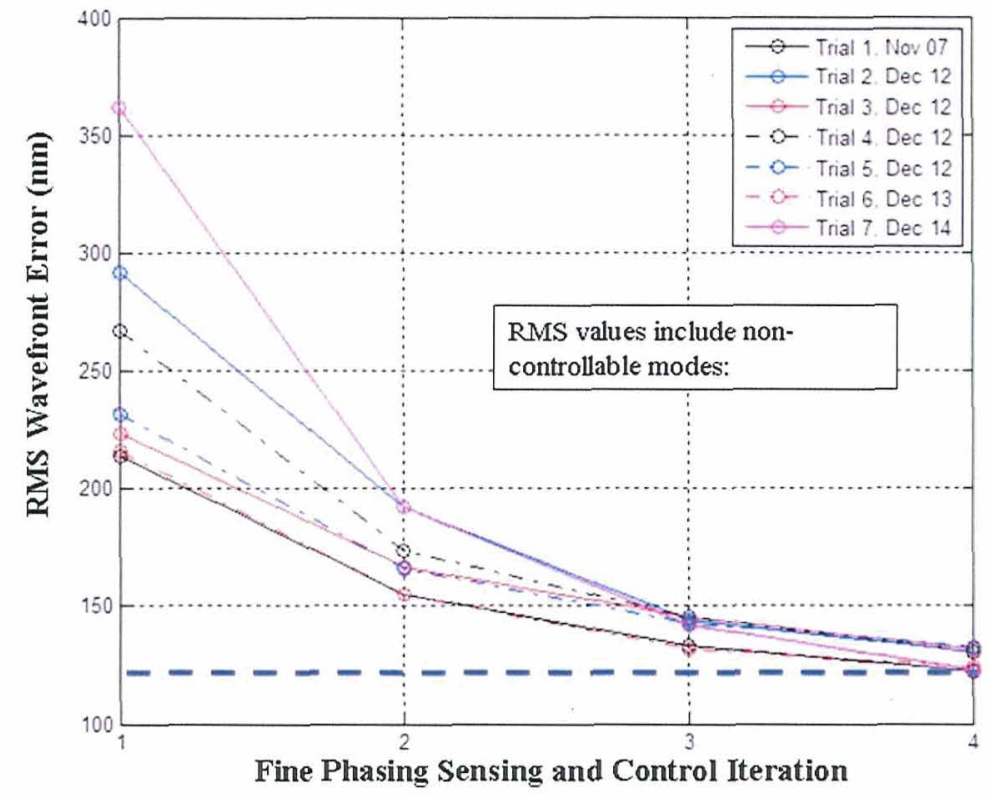

Fig. 15. Fine phasing convergence for seven independent tests. The dashed blue line at the bottom of the plot indicates the approximate theoretical minimum wavefront error.

\section{Fine-Phasing Repeatability}

In addition to the fine-phasing convergence shown in Fig. 15, fine phasing repeatability was examined independently by B. Dean (GSFC) and S. Acton (Ball Aerospace) using Commissioning data collected on Dec. 12, Dec. 13, and Dec. 14. These are the 6 data files listed in Table 6.

Table 6. Fine-Phasing Data Analyzed for Repeatability.

\begin{tabular}{|l|l|}
\hline Date (2006) & Designation \\
\hline Dec 12 & Phase 1 \\
\hline Dec 12 & Phase 2 \\
\hline Dec 12 & Phase 3 \\
\hline Dec 12 & Phase 4 \\
\hline Dec 14 & Phase 5 \\
\hline Dec 13 & Phase 6 \\
\hline
\end{tabular}

From these six data files, 15 unique differences were formed. The results show that the repeatability is within compliance of the RMS "median of the differences" requirement established by the mini-PIT peer review committee. 


\section{Multi-field Wavefront Sensing and Control}

A misaligned Secondary Mirror ( $\mathrm{X}$ and $\mathrm{Y}$ translation, $\mathrm{X}$ and $\mathrm{Y}$ tilt) creates wavefront errors measured at the center of the science FOV. During the fine-phasing process, the primary mirror is adjusted to compensate for these errors. Interrogating off-axis field points, however, may reveal the presence of large wavefront errors. Measuring and correcting these "field-dependent" errors is the goal of the MF process. This Section summarizes the TRL-6 multifield (MF) demonstration conducted on the TBT. The results show that the MF algorithm is capable of reducing the field dependency of the TBT relative to the TBT design nominal field dependency. ${ }^{52}$

Coordinated moves between the Field Steering Mirror (FSM) and the Auto-Collimating Flat (ACF) enable placement of any field point within roughly a $6 \times 12$ arc-minute FOV on the center of either the visible or IR cameras. The MF measurement process is accomplished by recording the wavefront at 4 corners of a rectangular field and then at the on-axis point (field center; see Fig. 16). Subtracting the onaxis wavefront from the off-axis wavefront results in a global wavefront difference that can be entirely characterized by focus and astigmatism. By comparing the measured wavefront differences to an optical model, the corrective SM moves that are required to

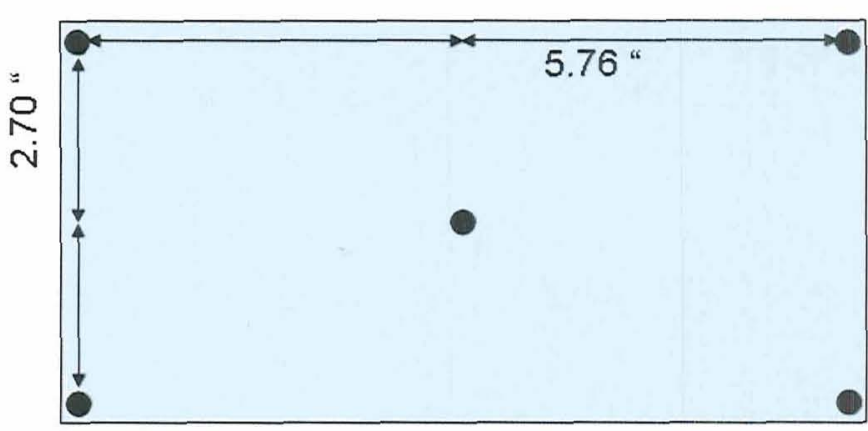

Fig. 16. Field points used in the MF measurements. The center field point is interrogated as well as the 4 corners at \pm 5.76 and \pm 2.70 arc-minutes.

eliminate the field dependency of the wavefront errors is obtained. Of course, once the SM corrections are applied, the PM must be adjusted to reestablish an acceptable wavefront error on axis. After these PM adjustments are made, there should be very little field dependence in the OTE wavefront error.

It should be noted that during the multi-field algorithm development process, ray-trace models showed that the fielddependency of the errors consisted of a strong linear function of the SM misalignment terms. ${ }^{53}$ Consequently, a single corrective action establishes the desired near-zero field dependent condition across the FOV. But it was also found that if the on-axis error compensation is incomplete, errors in the estimation of the corrective SM modes will occur. This simply implies that two or more iterations may be required to achieve adequate correction of the fielddependent errors.

\section{Multi-Field Tests with a Hartmann Sensor}

The deployment conditions used in the commissioning demonstration had large SM positioning errors and consequently, large field dependent errors. The first MF test was conducted with a simple Hartmann wavefront sensor, placed at a pupil image in the TBT Trunk Circuit. An example of a Hartmann sensor image is shown in Fig. 17.

The wavefront was measured at the 4 corner field points (Fig. 16) and at the center. The center wavefront was then subtracted from each corner and decomposed into focus and astigmatism terms. Corrective SM modes were estimated (from the linear model) and applied as corrections. The PM segments were also adjusted to remove segment-level tip and tilt errors.

The total correction applied to the SM along with the actual SM misalignments remaining after the single-field point commissioning process.

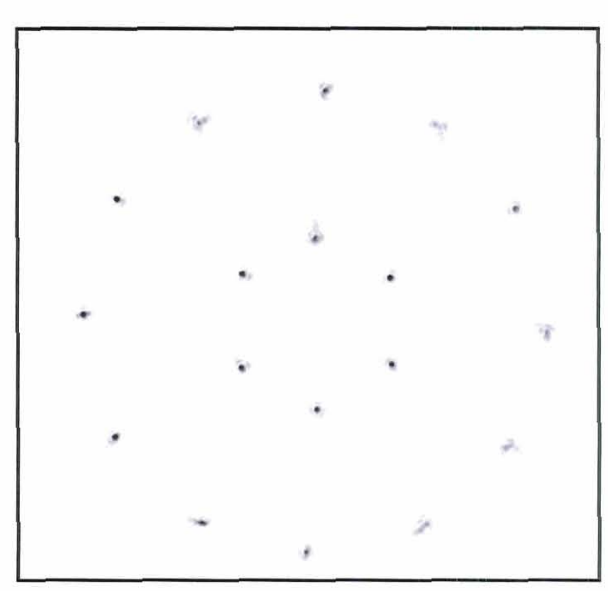

Fig. 17. Typical Hartmann Sensor Image. 
The corrections generated through the iterative multi-field process are accurate since we rely on a direct measurement of the MF errors. As can be seen, the original measurements of the TBT alignment (using a less accurate indirect technique) were off by several micro-radians in the $Y$ tilt axis.

Multi-Field Tests with Phase Retrieval

Having established the MF algorithm as a robust approach for correcting the SM position, the closed-loop test was repeated using phase retrieval to measure the wavefront errors at each field point. First, the TBT was returned to the post-commissioning state used in the previous test. Coarse phasing and single-field point fine-phasing were then applied. Immediately following the fine-phasing test, MF images were taken at each of the five field points. The wavefront was recovered from the images using the phase retrieval algorithm, discarding all but focus and astigmatism terms. The linear model was applied to estimate the corrective SM modes.

Having moved the SM, it was necessary to re-adapt the PM in modes higher than just tip and tilt. This was accomplished by going back to the Global Alignment commissioning step, and just correcting the PM clocking errors and ROC errors. Coarse phasing and single-field point fine-phasing was repeated, followed by a second acquisition of MF phase retrieval data; an analogous process is anticipated for flight. Then the phase retrieval images were analyzed as before, the SM modes estimated, and the correction applied. The Global Alignment commissioning step was repeated once again to correct the clocking and ROC errors. As before, this was followed by coarse phasing and fine-phasing, and MF phase retrieval data acquisition. This last set of MF images was analyzed and the MF wavefront errors calculated.

\section{Fine-Phasing Verification}

Upon completion of the fine-phasing commissioning step listed in Table 3, a Zygo GPI-XP ${ }^{54}$ interferometer was inserted into the beam path to measure the TBT optical wavefront. The phase retrieval and interferometer wavefront results were then compared for accuracy. The Zygo GPI-XP interferometer was taken as the "truth sensor," where it should be noted that the Zygo is checked for performance and calibration on a yearly cycle. The calibration work is performed at Ball Aerospace by Zygo field technical support staff, according to Zygo test procedures. The most recent calibration inspection occurred on September $15,2006 .{ }^{55}$ The only anomaly seen in the package is 4 to $5 \mathrm{~nm}$ RMS of fringe print-through noise. This contribution was identified as an artifact of the "set-up" made with the limited access available in the TBT installation. This artifact is a higher spatial frequency wavefront contribution and is not believed to have significantly affected the fine-phasing and interferometer comparison testing, as discussed below.

As discussed in the Section above, a complete end-end commissioning demonstration was conducted over a 1-week period starting in October 2006. Subsequently, and at the recommendation of the mini-PIT committee, seven additional fine-phasing commissioning demonstrations were completed on November 7, December 13 and 14 of 2006. These additional commissioning runs were completed to build confidence in the overall convergence and repeatability of the fine-phasing process.

The interferometer data used for the fine-phasing verification was collected on December 13 and 14 of 2006 . The data was collected immediately following completion of the fine-phasing process. The December 13 data files consisted of a multi-frame data set (14 interferometer readings). The December 14 data files also consisted of a multi-frame data set (12 interferometer readings). All data files of the multi-frame set were collected in immediate succession of the previous data capture. The Dec 13 interferometer data files were analyzed by Rick Lyon at GSFC. The Dec 14 data files were analyzed independently by Bruce Dean at GSFC.

An important consideration that must be factored into the comparison of the phase retrieval and interferometer views of the TBT is a fixed Non-Common-Path (NCP) contribution to the wavefront that results from slightly different views of the TBT as measured by the phase retrieval camera and the interferometer. The phase retrieval (PR) and interferometer (IF) wavefronts are thus related by the simple equation:

$$
P R_{\text {result }}=I F_{\text {result }}+N C P_{\text {term }} \text {. }
$$

Therefore, comparison of the phase retrieval and interferometer results must account for the NCP contribution through a calibration step. To characterize the NCP term, the beam path to the PM was blocked using a reference 
flat. Diversity defocus images were then collected and analyzed using the phase retrieval algorithm ${ }^{34}$ to determine the NCP term. The NCP term is dominantly astigmatism and the results of the comparison to the Dec 14 data are shown in Fig. 18.

In summary of the Dec 14 results, the fine-phasing result is shown on the far left of Fig. 18. The multi-frame average of the interferometer data is shown to the immediate right of the fine-phasing result (center left). The difference between the fine-phasing and interferometer result, labeled $\mathbf{A}$ in the figure, is shown in the next subfigure after decomposing each segment wavefront difference into a 15 term (order 4) orthogonal basis set (see Ref ${ }^{34}$ for more discussion of this basis set decomposition). The segment level decomposition has a filtering effect on the higher order and mid-spatial frequency segment wavefront errors. This contribution to the wavefront is noncontrollable since the mid-spatial frequency component does not correspond to the influence functions derived from the segment level opto-mechanical degrees of freedom. Therefore, the segment decomposition isolates the controllable degrees of freedom that were commanded during the fine-phasing commissioning process.

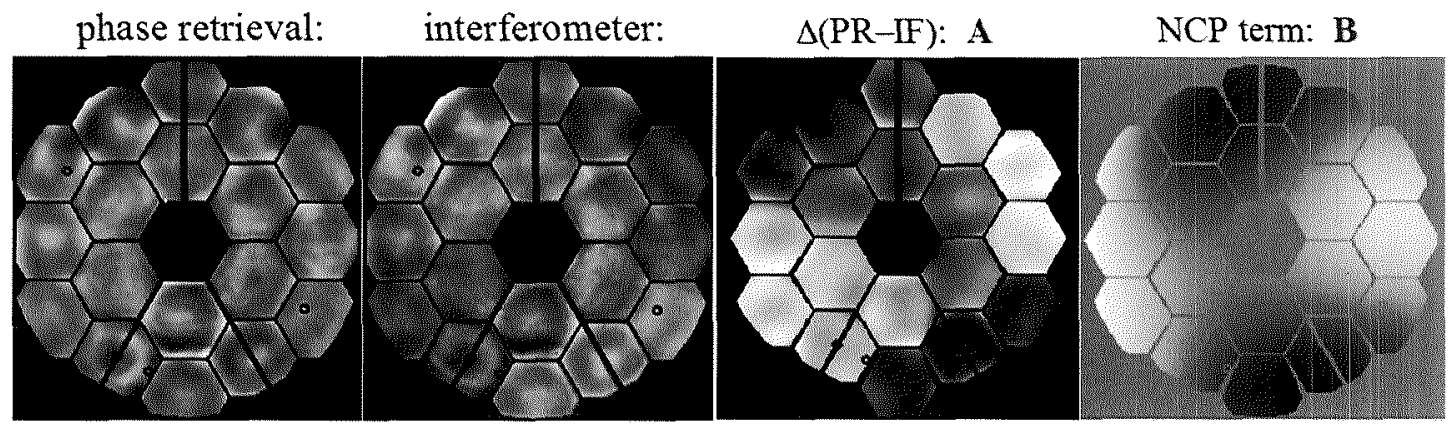

Fig. 18. Comparison between the Phase Retrieval and Interferometer Results. The NCP term is shown on the far right.

The NCP result is shown on the far right of Fig. 18 (labeled B) after masking with the TBT pupil (for consistency with the interferometer view of the TBT). Subtracting $\mathbf{A}$ from $\mathbf{B}$ then gives the error associated with the finephasing commissioning results in RMS double-pass wavefront error. This result is less than the TRL-6 RMS criteria and corresponds to using a full 15 term (order 4) basis set representation of the controllable modes. This is a conservative estimate in the sense that using a lower order representation of the controllable modes will give a smaller wavefront error (since higher order non-controllable modes are filtered out). In actuality, the controllable degrees of freedom of the TBT in these fine-phasing experiments were limited to just 5 controlled terms: piston, tip, tilt, astigmatism $x$ and astigmatism $y$.

A similar analysis was completed independently by Rick Lyon using the Dec 13 data set noting that in this particular comparison, the controllable degrees of freedom were based on a 9 term decomposition rather than the 15 term set used above to analyze the Dec 14 data. Based on the comments above, the 9 term decomposition more closely matches the actual controllable degrees of freedom in the fine-phasing experiment, and as a result, the error reported between the fine-phasing and interferometer results is correspondingly smaller.

\section{PSF Model vs. Measured}

The team performed an independent "sanity check" on the in-focus PSF. This was done by taking through focus point spread functions (PSFs) after the TBT had been aligned. These were then compared to a model of the PSF. The as-built error for the actual measurement included the secondary mirror alignment state at the time of the measurement. Other parameters such as drift are based on actual measurements made during earlier work. A comparison of the model result and the actual in-focus PSF is shown in Fig. 19. As can be seen on the figure, the model and actual data matched very well giving further confidence that the alignment process was successful. 


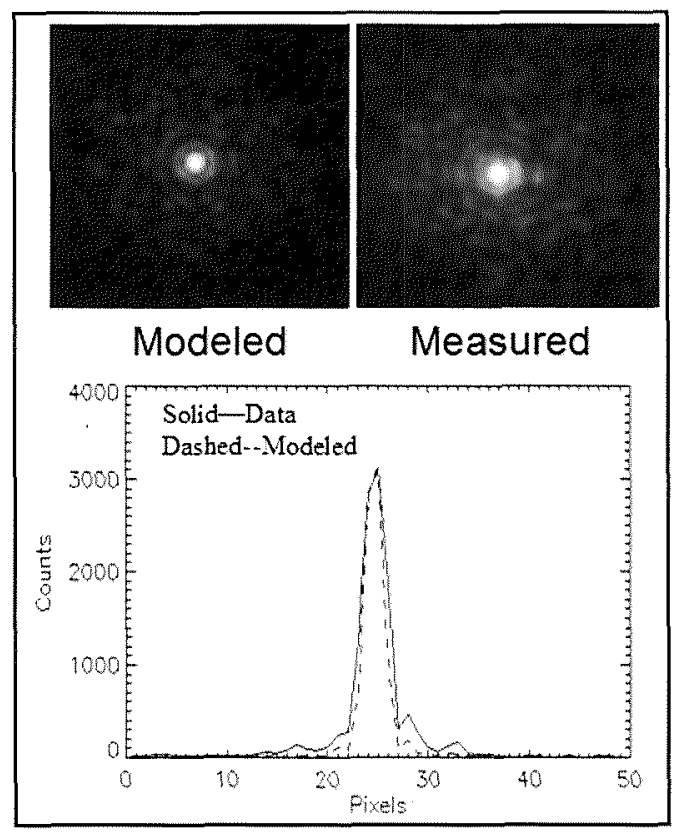

Fig. 19. Modeled and Measured TBT Point Spread Function

\section{SUMMARY AND DISCUSSION}

Early in the JWST program (pre-Phase A) the algorithm development relied heavily on modeling to demonstrate that WFSC could achieve the required tolerances using the specified control degrees of freedom. These early modeling efforts demonstrated that the image-based approach was a viable method for the JWST active optical control. Also during this pre-Phase A development, the fine-phasing algorithm approach for JWST leveraged from the HST phase retrieval results, and several lessons learned from the HST flight repair mission were incorporated into the JWST WFSC system architecture as discussed earlier in the "History and Prior Art" Section. After conclusion of the pre-Phase A program, development of the WFSC algorithms continued through direct hardware testing using various ground based telescopes and some flight instruments (Table 4). The technology was further validated in Phase-B with various Testbeds (Table 3). These WFSC Testbeds were built at GSFC and at Ball Aerospace (WCT, RA-6, and TBT), and were used to develop JWST-specific technologies to TRL 4 using 3 to 6 segment optical systems. In addition to contributing to early TRL development, these Testbeds helped to prove the overall robustness of the JWST WFSC approach in deployment scenarios where misalignments were significantly larger than the expected range of launch deployments. The coarse phasing process and DHS component were independently validated through two experiments on the inner 18 segments of the Keck Telescope. The results demonstrated that the specific coarse phasing approach to be used on JWST was at least as accurate as the existing Keck PCS camera. After development of a JWST scaled optical system Testbed Telescope (TBT), several key TRL-6 tasks were then validated including the complete "end-to-end" Observatory commissioning process. These commissioning steps are traceable to JWST performance levels from representative deployment misalignments using the TBT at a single-field point. The TBT has comparable control degrees of freedom to flight (segment piston, tip, tilt, ROC, clocking, $\mathrm{x} \& \mathrm{y}$ translation) and error budget requirements traceability. Also validated in this test program is the MF alignment capability with independent verification using a Shack-Hartmann sensor.

As part of these technology development efforts, several valuable "lessons learned" were gained. The first lesson learned by the team was that it is useful to use a second wavelength during initial single-field fine-phasing to assure there are no $2 \pi$ ambiguities in the final phased wavefront. One instance occurred where this was not the case but this was traced to an improper DHS calibration; so assuring that the DHS calibrations are correct is also a lesson learned. Another lesson learned included the development of a Hybrid Diversity technique for assuring that branch points and discontinuities in the segmented wavefront can be dealt with via the algorithms. ${ }^{34}$ The MF algorithm demonstration also yielded lessons learned including the fact that the MF approach to removing alignment 
ambiguities works as expected, and that the noise associated with the process is only that associated with the alignment terms used in the actual algorithm. This latter point means that the current JWST error budget approach for MF WFSC is somewhat conservative in that it doesn't separate out the alignment term noise (it uses the full WFSC noise). The final lesson learned is that it is important to perform sufficient phase retrieval iterations, and this is partly enabled by using a high speed digital signal processor. All of these lessons learned will be addressed as part of the plan forward for the WFSC algorithms.

In summary of the formal TRL- 6 compliance, five key technology elements have been validated for TRL-6. These include (1) development of the DHS component, (2) the development of the Coarse Phasing process that incorporates the DHS, (3) end to end single-field point Fine-Phasing, (4) independent validation of the Fine-Phasing performance, and (5) demonstration and validation of the MF point Fine-Phasing process. As a result of these efforts, all aspects of end-to-end WFSC for JWST have been demonstrated either at the component level (for the DHS element) or on the Testbed Telescope (TBT). Moreover, the TBT had traceable performance and degrees of freedom to JWST, and even had higher vibration and worse thermal stability than expected in flight. Each algorithm was tested many times individually and as part of the end-to-end testing, all were found to be robust. Backups exist within the architecture including a long wavelength grism and a pupil imaging lens. The material in this report was presented to the WFSC Mini-PIT peer review panel and they concurred that TRL-6 had been met on January $4^{\text {th }}$, 2007. The JWST team is therefore highly confident that the flight algorithms will ultimately be successful on-orbit and, as documented in this report, have demonstrated that the flight algorithms have met the TRL- 6 criteria of being tested in a relevant environment.

\section{REFERENCES}

1. P. A. Sabelhaus, D. Campbell, M. Clampin, J. Decker, M. Greenhouse, A. Johns, M. Menzel, R. Smith, and P. Sullivan, "An overview of the James Webb Space Telescope (JWST) project," Proc. SPIE, Vol. 5899, (2005).

C. B. Atkinson, S. C. Texter, L. D. Feinberg, and R. A. M. Keski-Kuha, "Status of the JWST optical telescope element," Proc. SPIE, these proceedings (2006).

2. J. Gardner, J. Mather, M. Clampin, R. Doyon, M. Greenhouse, H. Hammel, J. Hutchings, P. Jakobsen, S. Lilly, K. Long, J. Lunine, M. McCaughrean, M. Mountain, J. Nella, G. Rieke, M. Rieke, H. Rix, E. Smith, G. Sonneborn, M. Stiavelli, H. Stockman, R. Windhorst, G. Wright, "Science with the James Webb space telescope," Proceedings of SPIE, Vol 6265, (2006).

3. JWST-RQMT-000634, “JWST Mission Requirements Document," Revision N (Aug 30, 2006).

4. D. J. Schroeder, "Astronomical Optics," New York: Academic Press (1987).

5. J. Nella, C. Atkinson, A. Bronowicki, E. Bujanda, A. Cohen, D. Davies, M. Mohan, J. Pohner, P. Reynolds, S. Texter, D. Simmons, D. Waldie, R. Woods, R. Lynch, R. Lundquist, M. Menzel, B. Smith, P. Sullivan, P. Atcheson, P. Lightsey, "James Webb Space Telescope (JWST) Observatory Architecture and Performance," AIAA-2004-5986 American Institute of Aeronautics and Astronautics (2004). http://www.stsci.edu/jwst/docs/presentations/JwstObservatoryArchitectureAndPerformance 3318.pdf.

6. S. E. Kendrick, D. Chaney, and R. J. Brown, "Optical characterization of the beryllium semi-rigid AMSD mirror assembly," Proc. SPIE, Vol 5180, 180-187 (2004).

7. T. B. Parsonage, "JWST beryllium telescope: material and substrate fabrication," Proc. SPIE 5494, 39 (2004).

8 The telescope is designed for operation over a temperature range of 30-60 K.

9. A.R. Contos, et. al, "Wavefront Sensing \& Control Requirements Allocation Document (DRD OTE-14a, JWST-RQMT-002017)," Ball Aerospace \& Technologies Corp. (2006). 
10. M. Albanese, A. Wirth, A. Jankevics, T. Gonsiorowski, C. Ohara, F. Shi, M. Troy, G. Chanan, S. Acton, "Verification of the James Webb Space Telescope coarse phase sensor using the Keck Telescope," Proc. SPIE 6265 (2006).

11. TRL-6 Definition: System/subsystem model or prototype demonstration in a relevant environment (ground or space). See also:

J. C. Mankins, "Technology Readiness Levels: A White Paper," NASA, Office of Space Access and Technology, Advanced Concepts Office, April 6 (1995).

12. F. Shi, D. C. Redding, J. J. Green, and C. M. Ohara, "Performance of segmented mirror coarse phasing with a dispersed fringe sensor: modeling and simulations," Proc. SPIE 5487, 897 (2004)

13. F. Shi, C. M. Ohara, G. Chanan, M. Troy, D. C. Redding, "Experimental verification of dispersed fringe sensing as a segment-phasing technique using the Keck Telescope," Proc. SPIE 5489, 1061 (2004).

14. Jim Oschmann (BATC), Marty Huisjen (BATC), Scott Acton (BATC), Charlie Atkinson (NGST), Don Davies (NGST), Mark Clampin (GSFC), Bruce Dean (GSFC), Bill Hayden (GFSC), Dave Redding (JPL), Fang Shi (JPL), Brent Ellerbroek (Cal Tech/TMT), Scott Horner (LM), Matt Mountain (AURA/Gemini), Marcia Rieke (UA), Anand Sivaramakrishnan (AURA/StSci), Conrad Wells (ITT), "DHS / DFS Independent Design Review Summary and Disposition," Ball Systems Engineering Report, March, 2005.

15. J. H. Crocker, H. C. Ford, G. F. Hartig, and R. I. Jedrzejewski, "Optical performance of the Corrective Optics Space Telescope Axial Replacement (COSTAR)," Proc. SPIE, Vol. 2198, 1170 (1994).

16. J. R. Fienup, J. C. Marron, T. J. Schulz, and J. H. Seldin, "Hubble Space Telescope characterized by using phase retrieval algorithms," Appl. Opt. 32, 1747-1767 (1993).

J. E. Krist and C. J. Burrows, "Phase retrieval analysis of pre and post-repair Hubble Space Telescope images," Appl. Opt. 34, 4951-4964 (1995).

R. Lyon, P.E. Miller, and A. Grusczak, "Hubble Space Telescope Phase Retrieval: A Parameter Estimation," Proc. SPIE, Vol. 1567 (1991).

R. Lyon, J. Dorband, and J. Hollis, "Hubble Space Telescope Faint Object Camera Calculated Point-Spread Functions," Appl. Opt. 36, 1752-1775 (1997).

C. Roddier and F. Roddier, "Combined Approach to the Hubble Space Telescope Wave-Front Distortion Analysis," Appl. Opt. 32, 2992-3008 (1993).

17. A. Wissinger, "Sensing and Control for Large Optical Systems," Perkin Elmer Corp., refers to phase retrieval technique under development by Ed Siebert, from the Proc. of a Workshop: The Next Generation Space Telescope, held at the STScI, Sept 13-15, p. 232 (1989).

18. P. S. Davila, A. E. Lowman, M. E. Wilson, R. Boucarut, C. LeBoeuf, D. Redding, and E. Young, "Optical design of the developmental cryogenic active telescope testbed." Proc. SPIE 3356, 141 (1998).

19. C. W. Bowers, P. S. Davila, B. H. Dean, B. D. Perkins, M. E. Wilson, D. C. Redding, S. A. Basinger, D. Cohen, A. E. Lowman, F. Shi, L. A. Burns, M. W. Fitzmaurice, T. A. Norton, P. Petrone III, and J. Wheeler, "Initial test results from the Next Generation Space Telescope (NGST) wavefront sensing and control testbed (WCT)," Proc. SPIE 4013, 763 (2000).

20. P. D. Atcheson, T. C. Towell, P. C. Quigley, "Segmented aperture system wavefront error sensing and correction experiments at BATC," Proc. SPIE 3785, 38 (1999). 
T. C. Towell, P. D. Atcheson, "BATC multipurpose wavefront error sensing assembly," Proc. SPIE 3785 , 178 (1999).

B. Martin, P. D. Atcheson, P. C. Quigley, "BATC testbed segmented mirror design, assembly, and operation," Proc. SPIE 3785, 168 (1999).

P. D. Atcheson, P. C. Quigley, T. C. Towell, B. Martin, "Design of the BATC testbed for wavefront error sensing and correction," Proc. SPIE 3785, 121 (1999).

21. N. C. Gallagher and B. Liu, "Method for Computing Kineforms that Reduces Image-Reconstruction Error," Appl. Opt. 12, 2328-2335 (1973).

R. A. Gonsalves, R. Dumais, and P. Considine, "On Optimal Holographic Filters," Proc. SPIE, Vol. 45, 1974.

22. R. A. Gonsalves and P. Considine, "Phase Retrieval from Modulus Data," J. Opt. Soc. Am. 66, 961-964 (1976).

23. W. H. Southwell, "Wavefront Analyzer using a Maximum Likelihood Algorithm," J. Opt. Soc. Am. A3, 396-399 (1977).

24. R. A. Gonsalves, "Phase Retrieval and Diversity in Adaptive Optics," Optical Engineering, 21, 829-832 (1982).

25. J. R. Fiemup, "Iterative method applied to image reconstruction and to computer-generated holograms," Opt. Eng. 19(3), 297-305 (1980).

J. R. Fienup, "Phase retrieval algorithms: a comparison," Appl. Opt. 21, 2758-2769 (1982).

26. C. Roddier and F. Roddier, "Wavefront Reconstruction from Defocused Images and the Testing of Groundbased Optical Telescopes,” J. Opt. Soc. Am., A10, 2277-2287 (1993).

C. Roddier and F. Roddier, "Combined Approach to the Hubble Space Telescope Wave-Front Distortion Analysis," Appl. Opt. 32, 2992-3008 (1993).

J. R. Fienup, J. C. Marron, R. G. Paxman, T. J. Schulz, J. H. Seldin, and B. Thelen, "Image Inversion Analysis of the Hubble Space Telescope," in Final Report: JPL Contract 958892 on HST OTA Analysis, August 1991.

R. G. Lyon, "DCATT Comparative Wavefront Sensing and Optical Control Study Final Report," Feb. 22 (1999).

27. W. H. Press, S. A. Teukolsky, W. T. Vetterling, and B. P. Flannery, Numerical Recipes in C, Cambridge University Press, second ed. (1992).

28. Thomas Zielinski, Matthew Bolcar, and James R. Fienup, "Wavefront densing risk reduction," October 30 , 2006, University of Rochester, Institute of Optics, NASA Grant NNG05GK63A.

29. D. L. Misell, “A method for the solution of the phase problem in electron microscopy," J Phys., D6, L6-L9 (1973).

30. R. W. Gerchberg and W. O. Saxton, "Phase determination from image and diffraction plane pictures in an electron-microscope," OPTIK, 34, 275 (1971). 
R. W. Gerchberg and W. O. Saxton, "A practical algorithm for the determination of phase from image and diffraction plane pictures," OPTIK, 35, 237-246 (1972).

W.O. Saxton, "Computer techniques for image processing in electron microscopy" in Advances in Electronics and Electron Physics, Supplement 10, L Marton and C. Marton, eds. (Academic Press, New York, NY) (1978).

31. D. S. Acton, P. D. Atcheson, M. Cermak, L. K. Kingsbury, F. Shi, and D. C. Redding, "James Webb Space Telescope wavefront sensing and control algorithms," Proc. SPIE, Vol. 5487, 887 (2004).

32. D. Redding, S. Basinger, A. Lowman, F. Shi, P. Bely, R. Burg, and G. Mosier, "Wavefront sensing and control for a Next Generation Space Telescope," Proc. SPIE, Vol. 3356, 758-772 (1998).

33. G. Fornaro, G. Franceschetti, R. Lanari, and E. Sansosti, "Robust phase-unwrapping techniques: a comparison," J. Opt. Soc. Am. A 13, 2355 (1996)

34. B. H. Dean, D. L. Aronstein, J. S. Smith, R. Shiri, and D. S. Acton, "Phase retrieval algorithm for JWST flight and testbed telescope," Proc. SPIE 6265, 626511 (2006).

35. C. S. Clark and T. Jamieson, "NIRCam pupil imaging lens mechanism and optical design," Proc. SPIE, Vol. 5904 (2005).

36. The flight deployed Hubble pupil image was not available but had to be estimated using a few iterations of the iterative transform method. Details are discussed in the literature; see Ref. 25.

37. B. H. Dean and C. Bowers, "Diversity selection for phase-diverse-phase retrieval," J. Opt. Soc. Am. A 20 1490-1504 (2003).

38. L. A. Ryder and T. Jamieson, "Lens design for the Near Infrared Camera for the James Webb Space Telescope," Proc. SPIE, Vol. 5904, 590409 (2005).

L. G. Burriesci, "NIRCam instrument overview," Proceedings SPIE, Vol. 5904, 590403 (2005).

M. J. Rieke and R. C. Powell, "James Webb Space Telescope Program NIRCam Concept Study Report," Univ of Arizona, Jan 31 (2003).

Additional NIRCam information is available on the website: http://ircamera.as.arizona.edu/nircam/

39. L. K. Kingsbury, P. D. Atcheson, "JWST testbed telescope: a functionally accurate scaled version of the flight optical telescope element used to develop the flight wavefront sensing and control algorithm," Proc. SPIE 5487, 875 (2004).

40. J. W. Contreras and P. A. Lightsey, "Optical design and analysis of the James Webb Space Telescope: optical telescope element," Proc. SPIE, Vol. 5524, 30 (2004).

41. D.S. Acton, 05-JWST-0426, “Testbed Telescope Stability,” Ball Aerospace, March 2006.

42. W. Hayden, "TBT TRL-6 Test Requirements," NASA GSFC, Sept. 2006.

L. Feinberg, "WFSC Mini-PIT Telecon," WFSC Minipit Telecon Dec 21 Rev4a.ppt, NASA GSFC, Dec. $21,2006$.

43. 28 meters in double pass which includes the entire telescope as well as aft optics and trunk circuit. 
44. The term "focal-plane data" is generic in the sense that we acknowledge "out-of-plane" defocus intensity measurements required by focus-diverse-phase retrieval.

45. J. J. Green, B. H. Dean, C. M. Ohara, D. C. Redding, and Y. Zhang, "Target selection and imaging requirements for JWST fine-phasing," Proc. SPIE, Vol. 5487, 944 (2004)

46. B. Dean, M. Bolcar, "Phase Retrieval Estimation of OSIM+ISIM+MIRI Wavefront Errors," JWST OSIM Kickoff Meeting, Northrop Grumman Offices, Greenbelt, MD, Nov. 2, 2006.

47. Duncan Moore/Univ of Rochester/Chair, Jim Fienup/Univ of Rochester, Bob Gonsalves/Tufts, George Hartig/StScI, Chuck Bowers/GSFC Science representative, Rick Lyon/GSFC, Gary Chanan/UC Irvine/Keck (for Keck Coarse Phasing), John Mangus/SAIC/IRT representative.

48. http://www.aoainc.com/index.html

49. D. S. Acton, (2007).

50. D. S. Acton, T. Towell, J. Schwenker, J. Swensen, D. Shields, E. Sabatke, L. Klingemann, A. R. Contos, B. Bauer, K. Hansen, P. Atcheson, D. Redding, F. Shi, S. Basinger, B. Dean, L. Burns, "Demonstration of the James Webb Space Telescope commissioning on the JWST testbed telescope," Proc. SPIE 6265, (2006).

51. Ball System Engineering Report BALL-LWST-WFSC-06-024, "Generation of test cases for TBT end-toend, single-field-point commissioning demonstrations," AGILE \# 2264343.

52. E. Sabatke, "Proposed TRL-6 Multifield demonstration and success criterion", BALL-JWST-WFSC-06025, Agile \# 2264729

53. E. Sabatke, "Investigation of FSM Multi-field technique on the Testbed Telescope", BALL-JWST-WFSC06-018, Agile \# 2257771.

54. http://www.zygo.com/?/products/gpi/

55. The file referenced below contains 17 pages documenting the procedure, Ball reference flat, Zygo standard flats and the test results:

Zygo Certification of Calibration, Certification \#: 100707, Customer Name: Ball Aerospace, Test Date: 09/15/2006, Desc: GPI-XP, Serial \#: 01-09-65937, Calibration Procedure: ZSP-OOO4

Certified Results: The Interferometer meets or exceeds its original factory performance specification. Next Calibration Due: 09/14/2007, Type of Test Performed: Fizeau Interference against Reference Standard

Reference Standards Used:

ZW -001, ZW -002. 